\title{
Integrating state-of-the-art in silico tools with molecular docking to predict the impact of the most deleterious amino acid substitutions on TRAPPC6A protein
}

\author{
Mohammed Baqur S. Al-Shuhaib ${ }^{1, *}$ and Jafar M. B. Al-Shuhaib ${ }^{2}$ \\ ${ }^{1}$ Department of Animal Production, College of Agriculture, Al-Qasim Green University, Al-Qasim, Babil 51001, Iraq \\ ${ }^{2}$ Babylon Directorate of Education, Ministry of Education, Babil 51001, Iraq
}

Trafficking Protein Particle Complex subunit 6A (TRAPPC6A) is an important molecule that is mainly involved in the transport of vesicles to the cis-Golgi membrane. Loss of function in this protein leads to a variety of severe disorders. The present study was conducted to prioritize the most deleterious effects of non-synonymous single nucleotide polymorphisms (nsSNPs) on TRAPPC6A protein. Two approaches were employed, sequence-based and structure-based, to predict which nsSNP has the most harmful effects on TRAPPC6A. Docking was performed to compare the ability of normal TRAPPC6A and its most deleterious mutants to bind with the corresponding receptor. All utilized in silico tools indicated highly damaging impacts of three nsSNPs, viz. W74C, G125S and G129D. Docking showed remarkable alterations in the atomic contact energy of TRAPPC6A binding with its receptor. The present finding provides a cost effective method for assessing the damaging effects of nsSNPs on TRAPPC6A, which may help in understanding the impact of this protein on neurodevelopmental disorders.

Keywords: Deleterious mutants, in silico tools, molecular docking, protein particle complex, single nucleotide polymorphism.

TRAFFICKING between intracellular compartments is mediated by vesicles and regulated by the highly conserved molecules. These molecules are encoded by the TRAPPC6A (Trafficking Protein Particle Complex subunit $6 \mathrm{~A})$ gene, which has recently attracted attention since emerging sets of mental disabilities and dysmorphic disorders are associated with the variations in this gene or its associated sequences ${ }^{1}$. The TRAPPC6A gene consists of six exons separated by five introns, with an open reading frame encoding up to 159 amino acids. The mature product of the TRAPPC6A gene is TRAPPC6A, also known as the TRS33 protein, which has a molecular

*For correspondence. (e-mail: mohammed79@agre.uoqasim.edu.iq) weight of $18 \mathrm{kD}$. This protein is part of a multi-subunit protein particle complex which is involved in several vesicle-mediated cellular transport events ${ }^{2}$. This complex is known as the TRAPP family, a highly conserved family of a large protein network consisting of 11 subunits in mammals, which is required for proper vesicular transport from the endoplasmic reticulum (ER) to the Golgi ${ }^{3,4}$. It is well established that TRAPPC6A participates in the regulation of these vesicular transportation routes via its binding with TRAPP family receptors ${ }^{5}$. TRAPPC6A deficiency also has a noticeable link with a series of disorders, such as non-verbal reasoning ${ }^{6}$, spondyloepiphyseal dysplasia $\operatorname{tarda}^{7}$, movement disorders ${ }^{8}$, muscular dystrophy ${ }^{9}$ and intellectual disability associated with speech problems ${ }^{10}$. Recently, a missense mutation within the TRAPPPC6A gene variations has been found to be associated with clinical syndromes with neurodevelopmental abnormalities and dysmorphic features ${ }^{11}$. Since several mutations detected in several subunits within this family have been strongly linked to many disorders, mutations in TRAPPC6A can account for susceptibility to various metabolic disorders. For this, understanding the functional and structural properties of this protein seems to be essential. However, the roles of mutations in complex diseases as pathogenic or neutral remain a difficult task and sometimes impossible because of expensive and time-consuming experiments ${ }^{12}$. Recently, many highthroughput innovations have been developed to predict the effects of non-synonymous single nucleotide polymorphisms (nsSNPs) on protein structure, stability and interaction by providing a more accurate indication for the deleterious nsSNPs on the analysed proteins ${ }^{13}$. Despite the main regulatory involvement of TRAPPC6A in many biological functions, no comprehensive study has been made to prioritize the most important deleterious nsSNPs of this highly crucial protein. Taking these data into consideration, an inclusive prediction of the impact of nsSNPs on TRAPPC6A is presented herein with particular focus on the role of these deleterious SNPs in altering binding with its receptor with the aid of molecular docking. 


\section{Materials and methods}

\section{Data retrieval}

The corresponding databases for the TRAPPC6A gene (gene ID: 79090) were analysed following their rsIDs in April 2020. All deposited SNPs were retrieved from the NCBI-dbSNP (https://www.ncbi.nlm.nih.gov/snp/) database. Subsequently, SNPs were verified using the ensemble genome browser 96 (https://asia.ensembl.org/index.html).

\section{Sequence-based predictions}

The effects of the retrieved nsSNPs on protein structure and biological activity were predicted using ten major and widely accepted in silico tools that rely only on the primary sequences of amino acid residues. The potentially deleterious effects of the nsSNPs were predicted using SIFT (Sorting Intolerant From Tolerant) ${ }^{14}$, PolyPhen (Polymorphism Phenotyping) ${ }^{15}$, REVEL (Rare Exome Variant Ensemble Learner) ${ }^{16}$, MetalR ${ }^{17}$, PROVEAN (PROtein Variation Effect ANalyser) ${ }^{18}$, PANTHER (Protein ANalysis THrough Evolutionary Relationships) ${ }^{19}$, SNAP2 (predicting functional effect of sequence variants) ${ }^{20}$, SUSPect (disease susceptibility-based SAV phenotype prediction) ${ }^{21}$, Mutation Assessor ${ }^{22}$ and $\mathrm{PhD}$ SNP (Predictor of human Deleterious Single Nucleotide Polymorphisms) ${ }^{23}$. The cumulative outcomes of utilized tools were assessed for each analysed nsSNP.

\section{Structure-based predictions}

The UniProtKB accession number for TRAPPC6A is O75865 and its NCBI reference sequence is NP 001257820.1. The 3D structure covers the full amino acid residues that were built using Rosetta-based 3D prediction tool $^{24}$. The efficiency of the generated 3D structure was assessed by QMEAN (Qualitative Model Energy ANalysis) ${ }^{25}$, and by the side chain parameters and psi/phi Ramachandran plot of the PROCHECK $\operatorname{server}^{26}$ (Supplementary Material 1). Ten different structure-based predictions were also done using ten well-known in silico tools, including I-Mutant2 (ref. 27), iStable (Integrated predictor for protein Stability change upon single mutation) ${ }^{28}$, mCSM (mutation Cut-off Scan Matrix) ${ }^{29}, \mathrm{SDM}^{30}$, DUET $^{31}$, MAESTRO (Multi AgEnt STability pRedictiOn upon point mutations) ${ }^{32}$, STRUM (STRucture-based stability change prediction Upon single-point Mutation ${ }^{33}$, Mupro $^{34}$, SNPs\&Go ${ }^{3 \mathrm{~d}}$ (predicting disease associated variations using GO terms $)^{35}$ and DynaMut ${ }^{36}$.

\section{In-depth prediction of the most damaging nsSNPs}

The entirely deleterious nsSNPs in both sequence-based and structure-based predictions were considered for fur- ther analyses. The potential of these entirely deleterious nsSNPs in altering the binding activity with ligands, proteins or receptors was assessed using COACH-D tool ${ }^{37}$. The evolutionary conservation status of these SNPs was analysed using ConSurf server ${ }^{38}$. To understand the effect of the most damaging SNPs on the 3D structure of TRAPPC6A, site-specific computational mutagenesis was conducted by mutating the native TRAPPC6A with its mutant forms using the Mutate script from Swiss model PDB viewer tool ver. 4.1.0 (ref. 39). The normal TRAPPC6A protein and its deleterious mutants were superimposed on each other using PyMol ver. 7.0.1 (Schrödinger, LLC.) Next, each 3D model was subjected to refinement to remove overhaul distorted geometries using the steepest descent energy minimization provided in the Gromacs parameter set ${ }^{40}$. More in-depth in silico analyses were performed for further confirmation of the deleterious role of each risky nsSNP on the modelling of the mutant proteins in each case. These experiments were performed using a recent release of the state-of-the-art CABS-flex 2.0 server (http://biocomp.chem.uw.edu.pl/ CABSflex2/), in which the molecular simulation was conducted for the wild-type and mutant forms of TRAPPC6A protein.

\section{Docking}

The most common corresponding receptors for binding with TRAPPC6A were retrieved from the STRING-10 server (https://string-db.org/) (Supplementary Material 2). The refined PDB forms of normal TRAPPC6A, as well as its most dangerous mutant forms, were subjected to molecular docking with the most relative receptors of TRAPPC1, TRAPPC2, TRAPPC2L and TRAPPC3 using a hierarchical blind docking protocol comprising patchdock tool ${ }^{41}$. The best docking solution was selected for further analysis using PyMol suite.

\section{Results}

\section{Data retrieval output}

A total of 4585 SNPs were retrieved from the NCBI$\mathrm{dbSNP}$ and verified from the ensemble genome browser 98, including six splice donor variants, six splice acceptor variants, ten stop gained, eleven frameshift variants, one stop loss, five start loss, one inframe insertion, five inframe deletion, one protein altering variant, 142 missense mutations (or nsSNPs), 52 stop region variants, 79 synonymous variants, 45 coding sequence variants, 17 5'-UTR, 108 3'-UTR, and 4204 intronic variants. All of the retrieved 142 nsSNPs of the TRAPPC6A protein were selected for the downstream in silico predictions. 


\section{RESEARCH ARTICLES}

\section{Sequence-based predictions}

The conducted in silico tools showed cumulative deleterious effects for four nsSNPs, viz. G41E, W74C, G125S and G129D on the TRAPPC6A structure and biological activity (Supplementary Material 3). Among the 142 retrieved nsSNPs, the total number of damaging nsSNPs predicted by each sequence-based tool was shown and annotated (Figure $1 a$ ). SNAP2 was shown to be the most sensitive tool for detecting deleterious effects of the analysed nsSNPs (107 deleterious nsSNP/142 total nsSNPs).

\section{Structure-based predictions}

Concordant results of these tools indicated entirely deleterious effects of seven nsSNPs, including E13G, L18P, G26S, R43H, G45S, L48V, L52P, F68L, V77A, F78L, M82R, L85M, Y93N, V94A, D97H, L103F, L104F, M106T, M106K, F122L, C124S, L126P, L127P, G129S, A130T, G135S, I136T, F152C (Supplementary Material 4) (Figure $1 b)$.

\section{In-depth analyses of the entirely damaging SNPS}

More analyses were conducted on the most deleterious W74C, G125S and G129D nsSNPs to explore the pattern of each to cause such drastic alterations in the mutant TRAPPC6A protein (Figure $1 c$ and Table 1). The ConSurf results revealed an extremely high conserved status of W74, G125 and G129 residues, with functional roles and buried positions within the evolutionary-based primary structure of TRAPPC6A (Supplementary Material 5). Results of the PTM-ssMP tool showed no posttranslational effects for these three residues. Except for W74C, the COACH-D tool predicted no participation of G125S and G129D nsSNPs in altering the binding activity of TRAPPC6A with other proteins (Supplementary Material 6). The superimposition of native TRAPPC6A and its three mutant models indicated close homology between the native template and the mutant models (Figure 2). This observation revealed a fair superimposition of the native TRAPPC6A with its three mutant forms. With regard to $\mathrm{W} 74 \mathrm{C}$ and $\mathrm{G} 125 \mathrm{~S}$, dramatic differences in polar interactions were observed between wild and mutant models in terms of distances and numbers (Figure $3 a$ and $b)$. Meanwhile, no addition or deletion was detected before and after mutation with G129D, and the only observed changes were restricted with altered distances between both states (Figure $3 c$ ). Further analyses were performed to assess the overall effect of each identified deleterious nsSNP on the 3D modelling of the TRAPPC6A structure. Though these risky nsSNPs were approximately positioned in the core of the targeted protein, their deleterious impacts were further demonstrated

via comparing the normal and mutant TRAPPC6A models. Results of explicit simulation, flexibility, structural clustering and dynamic protein fluctuation added another layer of confirmation regarding the ability of these SNPs to damage the 3D structure of the TRAPPC6A protein (Figure 4).

(a)
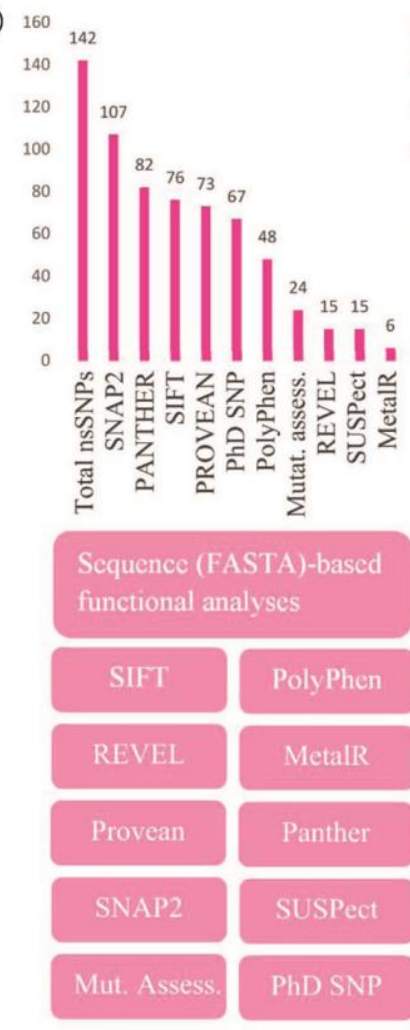

(b)

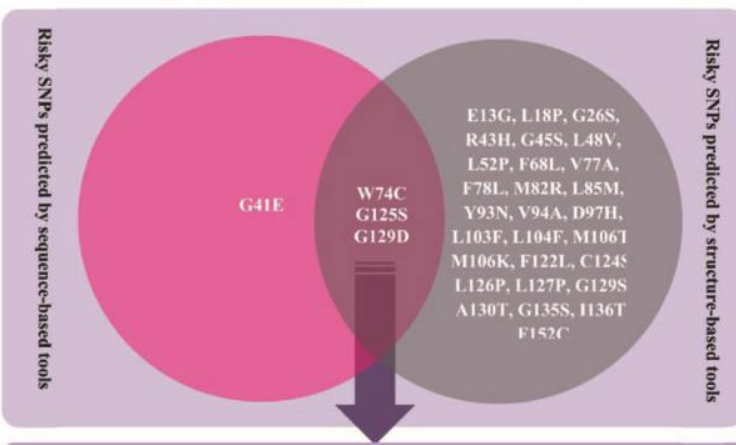

(c)

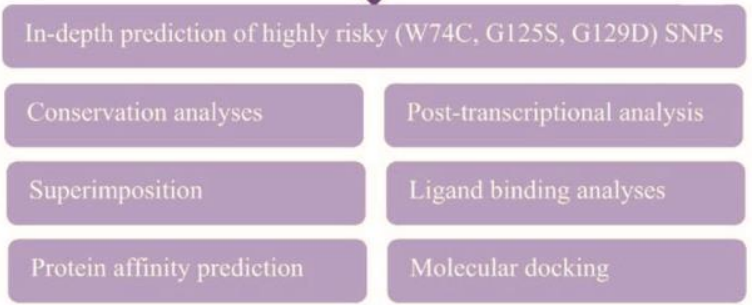

Figure 1. The in silico workflow of the present approach is used for predicting the most deleterious missense variants in the TRAPPC6A protein. $\boldsymbol{a}$, The main in silico predictions used and their sensitivity for prediction. $\boldsymbol{b}$, Vein diagram showing the most deleterious missense single nucleotide polymorphisms (SNPs). $\boldsymbol{c}$, The downstream in-depth in silico prediction for the identified deleterious SNPs. 


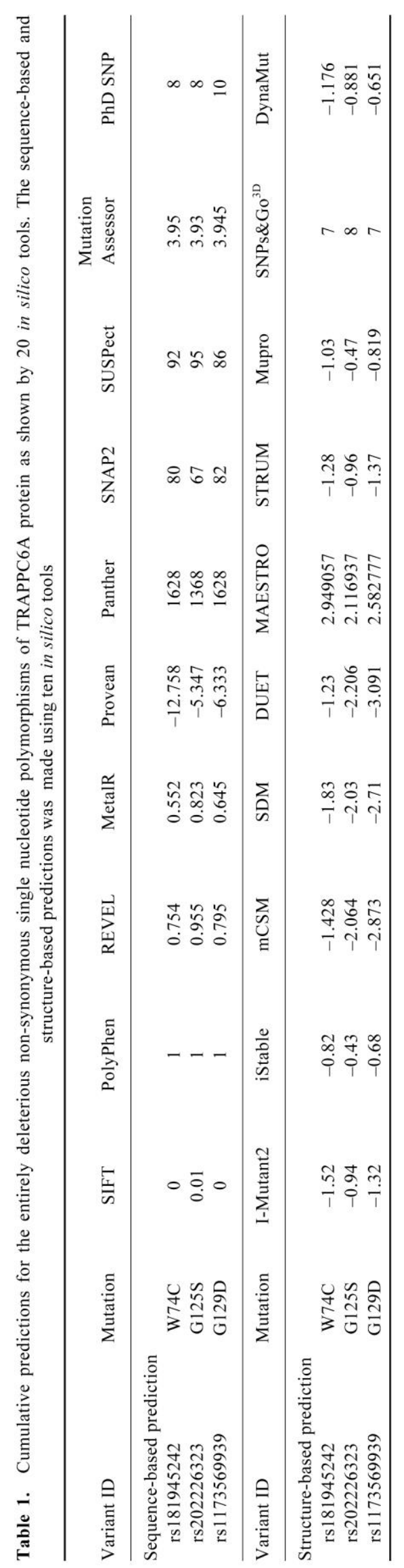



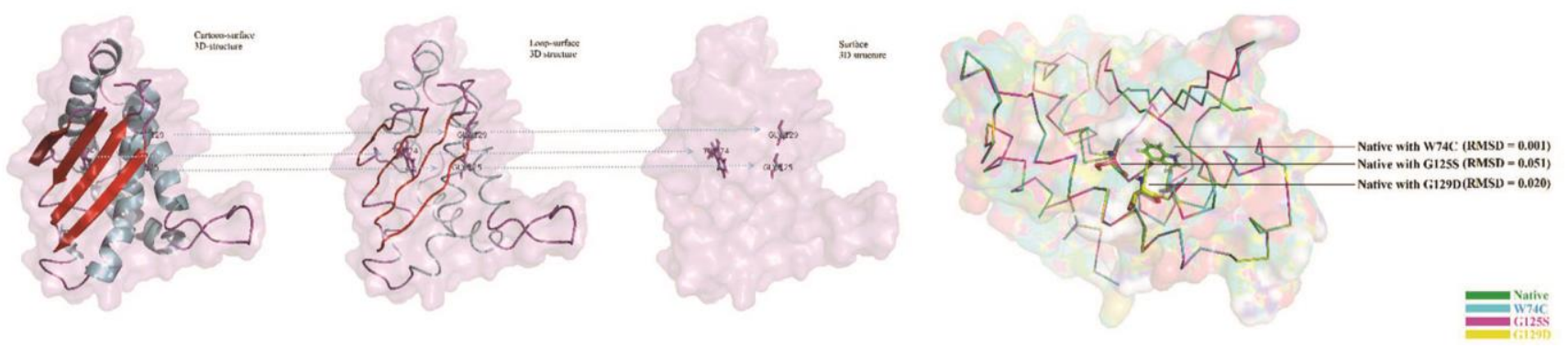

Figure 2. The main functional domains within TRAPPC6A (left) and spatial superimposition (right) of the native TRAPPC6A and the most deleterious mutants predicted in this study. The green, blue, magenta, and yellow colours refer to wild type and mutant forms res pectively.

(a)

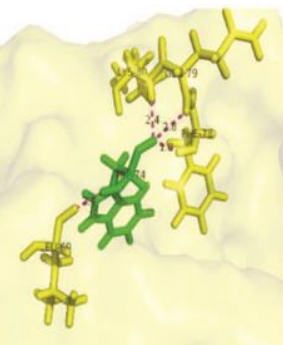

(b)

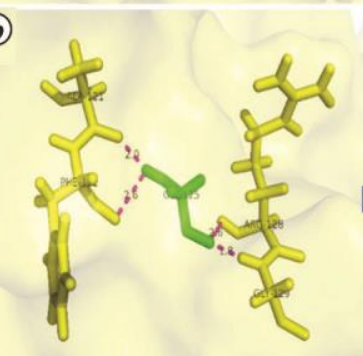

(c)

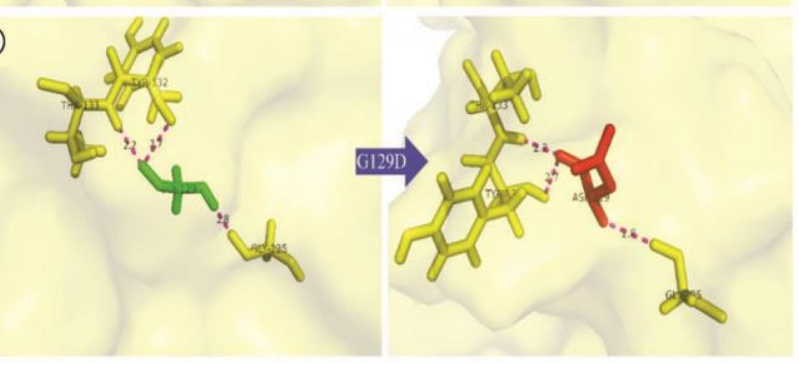

Figure 3. Alteration in polar interactions of the native TRAPPC6A and its W74C (a), G125S (b) and G129D ( $\boldsymbol{c}$ ) mutant forms in terms of the most deleterious amino acid residues with vicinal units before and after mutation.

\section{Docking with the close TRAPPC receptors}

The molecular docking between normal TRAPPC6A and its three risky mutant forms with TRAPPC1, TRAPPC2, TRAPPC2L and TRAPPC3 was conducted to identify the extent of variation in the overall ligand-receptors interaction before and after mutation. Analysis of interaction energies revealed a variety of roles for W74C, G125S and 9D models in inducing noticeable changes in the
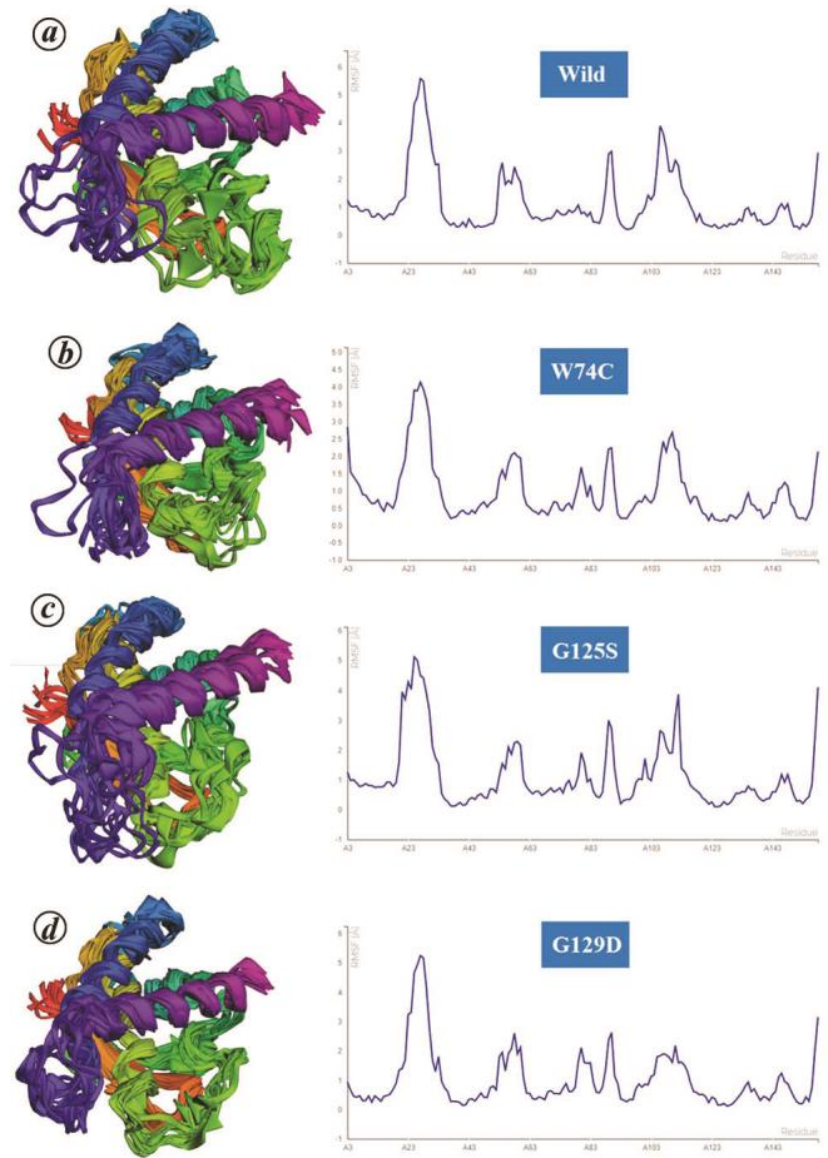

Figure 4. Aggregation propensity simulation and fluctuation plots for the wild-type TRAPPC6A and its deleterious models. $\boldsymbol{a}$, Wild-type protein. $\boldsymbol{b}-\boldsymbol{d}$, Deleterious W74C (b), G125S (c) and G129D (d) models. RMSF, Root mean square fluctuation.

binding of the wild TRAPPC6A with the other close TRAPPC receptors (Table 2).

\section{Discussion}

In the present study, a comprehensive in silico approach was used to determine the most deleterious missense 


\section{RESEARCH ARTICLES}

Table 2. Prediction of docking alteration in the binding TRAPPC6A and its most deleterious nsSNPs, L13P and G104S with the closest receptors in TRAPP-family members

\begin{tabular}{lllll}
\hline Docking complex & \multicolumn{1}{c}{ Wild-type } & \multicolumn{1}{c}{ W74C } & G125S & G129D \\
\hline TRAPPC6A-TRAPPC1 & Score (13978) & Score (14814) & Score (14256) & Score (14530) \\
& ACE2 (-142.47) & ACE2 (210.81) & ACE2 (197.35) & ACE2 (377.56) \\
TRAPPC6A-TRAPPC2 & Score (13294) & Score (13492) & Score (13922) & Score (13870) \\
& ACE2 (341.37) & ACE2 (342.98) & ACE2 (289.09) & ACE2 (296.04) \\
TRAPPC6A-TRAPPC2L & Score (12878) & Score (12324) & Score (14098) & Score (12556) \\
& ACE2 (482.45) & ACE2 (-47.99) & ACE2 (293.41) & ACE2 (268.24) \\
TRAPPC6A-TRAPPC3 & Score (15140) & Score (14734) & Score (15480) & Score (15262) \\
& ACE2 (365.17) & ACE2 (-86.49) & ACE2 (138.85) & ACE2 (113.51) \\
\hline
\end{tabular}

SNPs of TRAPPC6A using two sets of computational tools based on a variety of algorithms; sequence-based and structure-based tools. The necessity to determine whether each nsSNP having neutral or deleterious consequences is mandatory to prioritize the most damaging SNPs in many cases ${ }^{42,43}$. To obtain better assessment of the TRAPPC6A protein impacted by missense mutations, the retrieved nsSNPs were analysed using two different sets of in silico tools - sequence-based and structurebased. In the case of sequence-based tools, the input data were FASTA sequences of the TRAPPC6A protein. By comparing the cumulative computations of these methods, the degree of severity of the damaging consequences of each particular nsSNP was verified. Though each employed tool is commonly used to predict the impact of each variant on both protein structure and function ${ }^{14}$, at least four in silico tools are necessary to validate the sensitivity of each prediction ${ }^{44}$. Therefore, each prediction was assisted by other comparable prediction tools by analysing the effect of these nsSNPs on the biological activity of TRAPPC6A. SIFT ${ }^{14}$, PolyPhen ${ }^{15}$, REVEL ${ }^{16}$, MetalR ${ }^{17}$ and Provean ${ }^{18}$ were utilized to predict the effect of an nsSNP on both structure and function of the TRAPPC6A protein using straightforward and comparative algorithms. The functional validation of nsSNPs predicted by these tools was confirmed by Panther ${ }^{19}$, SNAP2 ${ }^{20}$, SUSPect ${ }^{21}$, mutation assessor ${ }^{22}$ and PhD SNP ${ }^{23}$ tools, which were used to classify SNPs by their evolutionary relationship, molecular functions and interaction with other proteins in their corresponding amino acid sequences. Though high efficiency of these predictions could be obtained, the use of FASTA data as the only input format may reduce the overall sensitivity and specificity of these results. Thus, to evaluate the effect of these deleterious nsSNPs on the TRAPPC6A protein upon mutation, another ten in silico tools were employed to prioritize these SNPs based on the 3D structure of the protein. Further assessment was obtained using I-Mutant2 (ref. 27), iStable ${ }^{28}, \mathrm{mCSM}^{29}, \mathrm{SDM}^{30}, \mathrm{DUET}^{31}$, MAESTRO $^{32}$, STRUM $^{33}$, Mupro $^{34}$, SNPs\&Go $^{3 D}$ (ref. 35), and DynaMut ${ }^{36}$ tools. However, all implemented computational programs have shown some discrepancies in the final effect of each nsSNP on the protein structure, func- tion and stability ${ }^{45}$. This observation is attributed to the differences in the algorithm that each software uses in its prediction. In this study, these fluctuated results were conquered by the cumulative utilization of many computation tools that were utilized to reduce the potential of errors and enhance the structural-functional grading of each prioritized SNP on the targeted protein ${ }^{46}$. Based on both sequence-based and structure-based approaches, three SNPs were detected with completely deleterious consequences, viz. W74C (rs181945242), G125S (rs202226323) and G129D (rs1173569939). For this, it is mandatory to get an insight into the mechanisms through which each one of these risky SNPs is based on to damage TRAPPC6A.

It can be inferred from the present evolutionary results that all three risky residues were not exposed to the surface and influenced through inducing structural effect on the TRAPPCA protein. Further confirmation for this structural role has come from the changes made in the distance and number of polar interactions after mutation. These amino acid substitutions may bring about several drastic changes in the 3D structure of the mutant TRAPPC6A. Since TRAPPC1, TRAPPC2, TRAPPC2L and TRAPPC 3 are the closest receptors of TRAPPC6A ${ }^{5}$, docking experiments were performed between the tertiary structures of normal TRAPPC6A and its three risky mutant models with these receptors. The analysis indicated the significant role of W74C, G125S and G129D in changing ACE score with clear conformational changes upon mutation. Though all these nsSNPs buried in positions away from the binding with the TRAPPC receptors, dramatic changes were observed in the binding with these receptors. This observation indicates an indirect role for these amino acid substitutions in inducing alterations in the 3D structure of the mutant TRAPPC6A in terms of its binding with TRAPP-interacting receptors ${ }^{5}$. Hence the altered binding may be associated with subsequent alteration in this interacting complex, which could lead to several cellular dysfunctions ${ }^{11}$. Thus, the clinical consequences of these amino acid substitutions deserve more attention due to the confirmed ability of the highly deleterious $\mathrm{W} 74 \mathrm{C}, \mathrm{G} 125 \mathrm{~S}$ and $\mathrm{G} 129 \mathrm{D}$ amino acid alterations to bring about major alterations in the TRAPPC6A 
architecture in such a way that it affects the subsequent interaction with TRAPP receptors. Therefore, it can be stated that these altered binding states between the deleterious TRAPPC6A models and their receptors appear to disrupt the downstream cascades of several versatile pathways associated with this interaction, with drastic consequences on several activities in the cell.

\section{Conclusion}

In this study we observed three highly damaging nsSNPs in TRAPPC6A, as revealed by 20 state-of-the-art in silico tools, based on the sequence or structure of the targeted TRAPPC6A. W74C, followed by G125S and G129D caused drastic conformational alterations in the binding of TRAPPC receptors. This study presents an in-depth interpretation for clinicians to estimate the impact of TRAPPC6-linked dysfunctions by knowing the type, grade and severity of each deposited nsSNP for this highly interesting molecule.

Conflict of interest: The authors declare no conflict of interest.

1. Sacher, M., Shahrzad, N., Kamel, H. and Milev, M. P., TRAPPopathies, an emerging set of disorders linked to variations in the genes encoding transport protein particle (TRAPP)associated proteins. Traffic, 2018, 20, 5-26.

2. Aridor, M. and Hannan, L. A., Traffic jams II: an update of diseases of intracellular transport. Traffic, 2002, 3, 781-790.

3. Kümmel, D., Oeckinghaus, A., Wang, C., Krappmann, D. and Heinemann, U., Distinct isocomplexes of the TRAPP trafficking factor coexist inside human cells. FEBS Lett., 2008, 582, 37293733.

4. Kim, J. J., Lipatova, Z. and Segev, N., TRAPP complexes in secretion and autophagy. Front. Cell Dev. Biol., 2016, 30, 20.

5. Scrivens, P. J., Shahrzad, N., Moores, A., Morin, A., Brunet, S. and Sacher, M., TRAPPC2L is a novel, highly conserved TRAPPinteracting protein. Traffic, 2009, 10, 724-736.

6. Hamilton, G. et al., Alzheimer's disease genes are associated with measures of cognitive ageing in the Lothian Birth Cohorts of 1921 and 1936. Int. J. Alzheimer's Dis., 2011, 2011, 505984.

7. Shaw, M. A. et al., Identification of three novel SEDL mutations, including mutation in the rare, non-canonical splice site of exon 4. Clin. Genet., 2003, 64, 235-242.

8. Bögershausen, N. et al., Recessive TRAPPC11 mutations cause a disease spectrum of limb girdle muscular dystrophy and myopathy with movement disorder and intellectual disability. Am. J. Hum. Genet., 2013, 93, 181-190.

9. Koehler, K. et al., A novel TRAPPC11 mutation in two Turkish families associated with cerebral atrophy, global retardation, scoliosis, achalasia and alacrima. J. Med. Genet., 2017, 54, 176185.

10. Brunet, S. and Sacher, M., In sickness and in health: the role of TRAPP and associated proteins in disease. Traffic, 2014, 15, 803818 .

11. Mohamoud, H. S. et al., A missense mutation in TRAPPC6A leads to build-up of the protein, in patients with a neurodevelopmental syndrome and dysmorphic features. Sci. Rep., 2018, 8, 2053.

12. Doss, C. G. P., Chakraborty, C., Chen, L. and Zhu, H., Integrating in silico prediction methods, molecular docking, and molecular dynamics simulation to predict the impact of ALK missense muta- tions in structural perspective. Biomed. Res. Int., 2014, 2014, 895831.

13. Al-Shuhaib, M. B. S., D76V, L161R, and C117S are the most pathogenic amino acid substitutions with several dangerous consequences on leptin structure, function, and stability. Egypt. J. Med. Hum. Genet., 2019, 20, 32.

14. Ng, P. C. and Henikoff, S., Predicting the effects of amino acid substitutions on protein function. Annu. Rev. Genomics. Hum. Genet., 2006, 22, 61-80.

15. Adzhubei, I. A. et al., A method and server for predicting damaging missense mutations. Nature Methods, 2016, 7, 248-249.

16. Ioannidis, N. M. et al., REVEL: an ensemble method for predicting the pathogenicity of rare missense variants. Am. J. Hum. Genet., 2016, 99, 877-885.

17. Dong, C. et al., Comparison and integration of deleteriousness prediction methods for nonsynonymous SNVs in whole exome sequencing studies. Hum. Mol. Genet., 2015, 24, 2125-2137.

18. Choi, Y., Sims, G. E., Murphy, S., Miller, J. R. and Chan, A. P., Predicting the functional effect of amino acid substitutions and indels. PLoS ONE, 2012, 7, e46688.

19. Tang, H. and Thomas, P. D., PANTHER-PSEP: predicting disease-causing genetic variants using position-specific evolutionary preservation. Bioinformatics, 2016, 32, 2230-2232.

20. Smigielski, E. M., Sirotkin, K., Ward, M. and Sherry, S., T2000 dbSNP: a database of single nucleotide polymorphisms. Nucleic Acids Res., 2000, 28, 352-355.

21. Yates, C. M., Filippis, I., Kelley, L. A. and Sternberg, M. J., SuSPect: enhanced prediction of single amino acid variant (SAV) phenotype using network features. J. Mol. Biol., 2014, 426, 26922701.

22. Reva, B., Antipin, Y. and Sander, C., Predicting the functional impact of protein mutations: application to cancer genomics. $\mathrm{Nu}$ cleic Acids Res., 2011, 39, e118.

23. Capriotti, E., Calabrese, R. and Casadio, R., Predicting the insurgence of human genetic diseases associated to single point protein mutations with support vector machines and evolutionary information. Bioinformatics, 2006, 22, 2729-2734.

24. Conchuir, S. O. et al., A web resource for standardized benchmark datasets, metrics, and Rosetta protocols for macromolecular modeling and design. PLoS ONE, 2015, 10, e0130433.

25. Waterhouse, A. et al., SWISS MODEL: homology modelling of protein structures and complexes. Nucleic Acids Res., 2018, 46, W296-W303.

26. Laskowski, R. A., MacArthur, M. W. and Thornton, J. M., PROCHECK: validation of protein structure coordinates. In International Tables of Crystallography, Vol. F, Crystallography of Biological Macromolecules (eds Rossmann, M. G. and Arnold, E.), Kluwer, Dordrecht, The Netherlands, 2001, pp. 722-725.

27. Capriotti, E., Fariselli, P. and Casadio, R., I-Mutant 2.0: predicting stability changes upon mutation from the protein sequence or structure. Nucleic Acids Res., 2005, 33, W306-W310.

28. Chen, C. W., Lin, J. and Chu, Y. W., iStable: off-the-shelf predictor integration for predicting protein stability changes. BMC Bioinform., 2013, 14, S5.

29. Pires, D. E. V., Ascher, D. B. and Blundell, T. L., mCSM: predicting the effects of mutations in proteins using graph-based signatures. Bioinformatics, 2012, 30, 335-342.

30. Worth, C. L., Preissner, R. and Blundell, T. L., SDM - a server for predicting effects of mutations on protein stability and malfunction. Nucleic Acids Res., 2012, 39, W215-W222.

31. Pires, D. E., Ascher, D. B. and Blundell, T. L., DUET: a server for predicting effects of mutations on protein stability using an integrated computational approach. Nucleic Acids Res., 2014, 42, W314-W319.

32. Laimer, J., Hofer, H., Fritz, M., Wegenkittl, S. and Lackner, P., MAESTRO - multi agent stability prediction upon point mutations. BMC Bioinform., 2015, 16, 116. 
33. Quan, L., Lv, Q. and Zhang, Y., STRUM: structure-based prediction of protein stability changes upon single-point mutation. Bioinformatics, 2016, 32, 2936-2946.

34. Cheng, J., Randall, A. and Baldi, P., Prediction of protein stability changes for single site mutations using support vector machines. Proteins, 2006, 62, 1125-1132.

35. Capriotti, E., Calabrese, R., Fariselli, P., Martelli, P. L., Altman, R. B. and Casadio, R., WSSNPs\&GO: a web server for predicting the deleterious effect of human protein variants using functional annotation. BMC Genomics, 2013, 14(Suppl. 3), S6.

36. Rodrigues, C. H., Pires, D. E. and Ascher, D. B., DynaMut: predicting the impact of mutations on protein conformation, flexibility and stability. Nucleic Acids Res., 2018, 46(W1), W350-W355.

37. Wu, Q., Peng, Z., Zhang, Y. and Yang, J., COACH-D: improved protein-ligand binding sites prediction with refined ligand-binding poses through molecular docking. Nucleic Acids Res., 2018, 46, W438-W442.

38. Ashkenazy, H., Erez, E., Martz, E., Pupko, T. and Ben-Tal, N., ConSurf 2010: calculating evolutionary conservation in sequence and structure of proteins and nucleic acids. Nucleic Acids Res., 2010, 38, W529-W533.

39. Johansson, M. U., Zoete, V., Michielin, O. and Guex, N., Defining and searching for structural motifs using DeepView/SwissPdbViewer. BMC Bioinform., 2012, 13, 173.

40. Van Gunsteren, W. F., Billeter, S. R., Eising, A. A., Hunenberger, P. H. and Kruger, P., Biomolecular Simulation: The GROMOS96 Manual and User Guide, vdf Hochschulverlag AG an der ETH Zurich and BIOMOS b.v, Zurich, Switzerland, 1996.
41. Schneidman-Duhovny, D., Inbar, Y., Nussinov, R. and Wolfson, H. J., PatchDock and SymmDock: servers for rigid and symmetric docking. Nucleic Acids Res., 2005, 33, W363-W367.

42. Al-Shuhaib, M. B. S., Deleterious amino acid substitutions with a series of putative damaging effects on egg components are revealed in the ovalbumin gene family; an in silico approach. Nova Biotechnol. Chim., 2019, 18(2), 1-9.

43. Anand, P. P., Computational modelling of human sarcomeric telethonin protein and predicting the functional effect of missense single nucleotide polymorphism. Curr. Sci., 2019, 117, 638-648.

44. Al-Shuhaib, M. B. S., Al-Kafajy, F. R. and Al-Jashami, G. S., A computational approach for explaining the effect of the $\mathrm{prl}$ gene polymorphism on prolactin structure and biological activity in Japanese quails. Anim. Biotechnol., 2019, 29, 1-9.

45. Sarhan, R. S., Hashim, H. O. and Al-Shuhaib, M. B. S., The Gly152Val mutation possibly confers resistance to beta-lactam antibiotics in ovine Staphylococcus aureus isolates. Open Vet. J., 2019, 9(4), 339-348.

46. Mustafa, K. M., Ewadh, M. J., Al-Shuhaib, M. B. S. and Hasan, H. G., The in silico prediction of the chloroplast maturase $\mathrm{K}$ gene polymorphism in several barley varieties. Agriculture (Pol'nohospodárstvo), 2019, 64(1), 3-16.

Received 18 June 2020; revised accepted 23 October 2020

doi: $10.18520 / \mathrm{cs} / \mathrm{v} 120 / \mathrm{i} 2 / 398-405$ 